\title{
Otimização das condições fermentativas de massas para a produção de pães tipo forma utilizando a medotologia de superfície de resposta
}

\author{
R. A. ZAMBELLI ${ }^{1}$, S. C. P. de MELO' ${ }^{1}$, L. I. F. PINTO ${ }^{1}$, D. L. BRASIL ${ }^{1}$, G. K. PINHEIRO ${ }^{1}$ \\ e D. F. PONTES ${ }^{1}$. \\ ${ }^{1}$ Universidade Federal do Ceará, Departamento de Tecnologia de Alimentos. \\ E-mail para contato: Zambelli@alu.ufc.br
}

\begin{abstract}
RESUMO - O estudo tem como objetivo utilizar o delineamento composto central rotacional na otimização da condições fermentativas de massas para a produção de pães. Foram definidas como variáveis independentes: a quantidade de fermento biológico seco e sal, as quais foram adicionadas em níveis variando de $1,6 \%$ a $5 \%$ e $0 \%$ a $4 \%$, respectivamente. Através da metodologia de superfície de resposta foram avaliados os efeitos sobre as características físicas e fermentativas das massas e pães: fator de expansão máximo, volume produzido e específico, densidade e índice de expansão. De acordo com os resultados obtidos, o ensaio 2, incorporado com $4,16 \%$ de fermento e $1 \%$ de sal apresentou o maior volume específico $(4,41 \mathrm{~mL} / \mathrm{g})$ e o menor valor de densidade $(0,23 \mathrm{~g} / \mathrm{mL})$. Quanto maior à quantidade de sal empregada, independente da quantidade de fermento, houve redução nas capacidades de expansão das massas. As condições ótimas encontradas foram: incorporação de até $2 \%$ de sal e fermento biológico entre $3 \%$ e $5 \%$.
\end{abstract}

\section{INTRODUÇÃO}

As propriedas físicas dos pães são importantes parâmetros de qualidade, os quais são afetados com frequência por modificações em formulações e condições de processos, também dependendo dos métodos de produção do pão (Rózylo et al., 2014).

A levedura, Saccharomyces cerevisae, tem a função de agente de fermentação, que gera dióxido de carbono, sendo responsável pela estrutura estrutura aerada da massa. Entretanto, na produção de pães a ação da levedura não se limita apenas à produção de gás, também são responsáveis pelo sabor e afetar a reologia da massa (Palomba et al., 2011). Estudos recentes sugerem que o efeito da adição de fermento à massa de pãoe seu efeito na reologia da massa depende da produção de metabólitos específicos produzidos pela levedura, tais como o etanol e o ácido succínico (Callejo, 2011). Katina et al. (2010) afirmam que o estado fisiológico das células de levedura podem afetar a produção dos metabólitos e o processo fermentativo, ponto onde o sal pode interferir na ação do fermento.

De acordo com Ouerdane e Zóltan (2008), as células de levedura cultivadas na presença de $3 \%$ de sal obtiveram aumento na sua capacidade fermentativa do que as células cultivadas sem a presença do sal, entretanto, o mecanismo ainda não é claro. O sal desempenha um papel importante na produção do pão, uma vez que influencia o comportamento do glúten, reforça a estrutura da massa, diminui a produção de gás através da redução da atividade do fermento e melhora o sabor do pão. Lynch et al., (2009) mostraram que a redução de sal de 1,2\% a 0,3\% 
em pão de forma não teve efeito significativo na textura e sabor do produto, enquanto que a remoção completa do sal produziu pães de textura mais dura e com sabor mais azedo e de levedo. O sal pode funcionar como agente regulador do processo fermentativo, reduzindo a atividade do fermento e produzindo pães de menor qualidade no que diz respeito às suas caracetrísticas de expansão. Foi demonstrado por Gomez et al. (2008) que o tempo de fermentação afeta a firmeza inicial dos pães e a taxa de endurecimento do miolo durante o armazenamento em pães processados com farinha de trigo branca.

Diante do exposto, o estudo tem como objetivo utilizar o delineamento composto central rotacional na otimização das condições fermentativas de massas para a produção de pães tipo forma, variando as concentrações de fermento biológico seco e sal empregadas.

\section{METODOLOGIA}

\subsection{Delineamento Experimental}

O desenvolvimento das formulações de pães tipo forma foi realizado a partir de uma formulação padrão, cuja composição é: $100 \%$ de farinha de trigo; $55-60 \%$ de água, $10 \%$ de Gordura Vegetal Hidrogenada; 5\% de açúcar, além do fermento biológico seco e sal, que são as variáveis independentes deste estudo.

$\mathrm{Na}$ tabela 1 são apresentados as faixas de valores codificados e reais utilizados para os ensaios e na tabela 2 é apresentada a matriz do planejamento.

Tabela 1 - Variáveis e níveis do planejamento experimental completo $2^{2}$

\begin{tabular}{|c|c|c|c|c|c|}
\hline \multirow[b]{2}{*}{ Variáveis Independentes } & \multicolumn{5}{|c|}{ Níveis codificados e reais das variáveis independentes } \\
\hline & $-\alpha=-1,41$ & -1 & $\mathbf{0}$ & +1 & $+\alpha=+1,41$ \\
\hline Fermento Biológico (g) & 5 & 7,5 & 10 & 12,5 & 15 \\
\hline Sal $(\mathbf{g})$ & 0 & 3 & 6 & 9 & 12 \\
\hline Fermento Biológico (\%) & $1,6 \%$ & $2,5 \%$ & $3,3 \%$ & $4,1 \%$ & $5 \%$ \\
\hline Sal $(\%)$ & $0 \%$ & $1 \%$ & $2 \%$ & $3 \%$ & $4 \%$ \\
\hline
\end{tabular}

*Em relação a farinha de trigo (base 100).

Tabela 2 - Matriz do delineamento experimental com valores codificados e reais

\begin{tabular}{ccccc}
\hline Ensaios & $\begin{array}{c}\text { Fermento } \\
\text { Biológico }\end{array}$ & Sal & $\begin{array}{c}\text { Fermento } \\
\text { Biológico }(\mathbf{g})\end{array}$ & Sal (g) \\
\hline $\mathbf{1}$ & -1 & -1 & 7,5 & 3 \\
$\mathbf{2}$ & +1 & -1 & 12,5 & 3 \\
$\mathbf{3}$ & -1 & +1 & 7,5 & 9 \\
$\mathbf{4}$ & +1 & +1 & 12,5 & 9 \\
$\mathbf{5}$ & $-1,41$ & 0 & 5 & 6 \\
$\mathbf{6}$ & $+1,41$ & 0 & 15 & 6 \\
$\mathbf{7}$ & 0 & $-1,41$ & 10 & 0 \\
$\mathbf{8}$ & 0 & $+1,41$ & 10 & 12 \\
$\mathbf{9}$ & 0 & 0 & 10 & 6 \\
$\mathbf{1 0}$ & 0 & 0 & 10 & 6 \\
$\mathbf{1 1}$ & 0 & 0 & 10 & 6 \\
\hline
\end{tabular}


Para o desenvolvimento das formulações de pães tipo forma incorporadas com diferentes quantidades de fermento biológico e sal foi utilizado o Delineamento Composto Central Rotacional (DCCR). Foi aplicado um planejamento fatorial $2^{2}$ completo, totalizando 11 ensaios.

\subsection{Processamento dos Pães Tipo Forma}

Os ingredientes foram pesados em balança semi-analítica separadamente. Aplicou-se o método direto, onde todos os ingredientes são colocados simlutaneamente no início da etapa de mistura, com exceção do sal e água. Eles foram misturados em misturadora de escala semiindustrial durante 1 minuto em baixa velocidade para a homogeneização dos ingredientes, em seguida foi adicionada a água e misturada por 3 minutos em velocidade média, por último foi adicionado o sal e a massa foi misturada em alta velocidade por 6 minutos até o seu completo desenvolvimento. As massas foram divididas em porções de $250 \mathrm{~g}$ e moldadas na forma de elipses manualmente. Foram colocadas em fôrmas de folha galvanizada de ferro de chapa única para pão de forma sem tampa. Em seguida, colocadas em câmara de fermentação regulada a temperatura de $28{ }^{\circ} \mathrm{C} \pm 2{ }^{\circ} \mathrm{C}$ e $80 \%$ de umidade relativa, durante uma hora e trinta minutos. Ao final da fermentação, as massas foram assadas sem vapor durante 20 minutos a temperatura de $220^{\circ} \mathrm{C}$ em forno elétrico de lastro Continental Advance Turbo®. Os pães foram resfriados durante uma hora em temperatura ambiente.

\subsection{Avaliação Física das Massas e dos Pães Tipo Forma}

Avaliação do processo de fermentação pela técnica de perfil livre de expansão: A expansão das massas durante o processo de fermentação foi avaliada através do procedimento experimental de Perfil Livre de Expansão desenvolvido por Gabric et al. (2011), com modificações, onde as massas foram consideradas como elipses truncadas, conforme ilustra a figura 1, foram medidas, a cada 10 minutos, as dimensões com o auxílio de régua milimetrada simples. As massas foram colocadas em fôrmas de alumínio com dimensões de $30 \mathrm{~cm}$ x 30 $\mathrm{cm}$ para permitir a sua livre expansão sem restrições dimensionais.

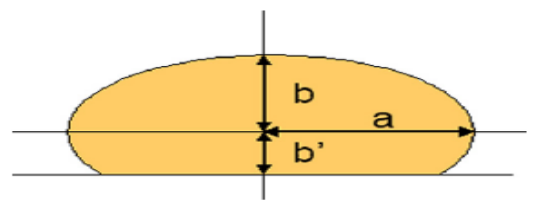

Figura 1 -Parâmetros de elipse truncada

O volume das massas para cada tempo de medição foi calculado com base na fórmula da elipse e sua rotação em torno do eixo y, conforme demonstra a equação 2 .

$V\left(c m^{3}\right)=\pi a^{2}\left(\frac{2 b}{3}+b^{\prime}-\frac{b^{3}}{3 b^{2}}\right)$

Onde: $\mathrm{a}=$ largura da elipse $(\mathrm{cm}) ; \mathrm{b}=$ altura da elipse - do centro a elipse superior $(\mathrm{cm})$; $b^{\prime}=$ altura da elipse - do centro para a parte inferior $(\mathrm{cm})$.

O fator de expansão do volume $\left(\mathrm{cm}^{3}\right)$ - VEF, foi calculado através da equação 3 .

$V E F=\frac{V-V_{o}}{V_{o}}$ 
Onde: $\mathrm{V}=$ Volume da massa ao longo do tempo de fermentação $\left(\mathrm{cm}^{3}\right) ; \mathrm{V}_{\mathrm{o}}=$ Volume incial da massa $\left(\mathrm{cm}^{3}\right)$.

Volume Produzido: Porções de massa de $15 \mathrm{~g}$, foram colocadas em provetas de $100 \mathrm{~mL}$ previamente esterilizadas para medição dos volumes das massas durante o processo de fermentação. Para o cálculo do volume produzido $(\Delta \mathrm{V})$ utilizou-se a subtração entre o volume final produzido, após uma hora e trinta minutos de fermentação e o volume inicial das massas na provetas, os resultados expressos em $\mathrm{mL}$.

Volume Específico: As massas dos pães foram determinadas em balança semi-analítica e expressa em gramas. $\mathrm{O}$ volume deslocado dos pães foi medido através de preenchimento de recipiente plástico e transparente com semente de painço expressando o resultado $\mathrm{mL}$. $\mathrm{O}$ volume específico foi calculado pela divisão do volume deslocado do pão (mL) pela sua massa (g), segundo método ${ }^{\circ} 72-10$ da AACC (1995).

Densidade: A densidade foi calculada através da relação inversa entre o volume deslocado e o peso da amostra assada.

Índice de Expansão: Para o cálculo do índice de expansão foi adaptada a medotologia utilizada por Silva et al. (2003) para pães de queijo. As massas foram moldadas na forma esférica para permitir as medições do diâmetro e da altura. com auxílio de régua milimetrada simples. O Índice de Expansão (IE) dos pães foi calculado através da equação 4:

Índice de Expansão $(I E)=\frac{\frac{\left(D_{p}+H_{p}\right)}{2}}{\frac{\left(D_{m}+H_{m}\right)}{2}}$

Onde: $\mathrm{D}_{\mathrm{p}}$ e $\mathrm{H}_{\mathrm{p}}=$ Diâmetro e altura dos pães após o forneamento (cm); $\mathrm{D}_{\mathrm{m}}$ e $\mathrm{H}_{\mathrm{m}}=$ Diâmetro e altura das massas moldadas $(\mathrm{cm})$.

\subsection{Análise Estatística}

A avaliação dos resultados dos parâmetros físicos no desenvolvimento das formulações através do delineamento composto central rotacional foi realizada por superfície de resposta e suas respectivas curvas de contorno após a análise de variância (ANOVA) e regressão, foi realizado teste de médias ao nível de $5 \%$ de significância. A análise foi realizada no programa STATISTICA 7.0.

\subsection{RESULTADOS E DISCUSSÃO}

A tabela 4 apresenta os valores obtidos para os parâmetros físicos dos pães tipo forma.

O E02, incorporado com 12,5 g de fermento biológico seco e $3 \mathrm{~g}$ de sal, apresentou o maior volume específico, com $4,41 \mathrm{~mL} / \mathrm{g}$, como consequência foi o ensaio que obteve o menor valor de densidade $(0,23 \mathrm{~g} / \mathrm{mL})$, a quantidade de sal incorporada não prejudicou as características físicas de expansão da massa, o fator de expansão máximo atingido foi de 2,12 $\mathrm{cm}^{3}$, o maior de todos os ensaios realizados. 
Tabela 4 - Parâmetros físicos dos pães tipo forma

\begin{tabular}{cccccccc}
\hline Ensaios $^{1}$ & $\begin{array}{c}\text { Fermento } \\
(\%)\end{array}$ & $\begin{array}{c}\text { Sal } \\
(\%)\end{array}$ & $\begin{array}{c}\text { Volume } \\
\text { Específico } \\
(\mathbf{m L} / \mathbf{g})\end{array}$ & $\begin{array}{c}\text { Densidade } \\
(\mathbf{g} / \mathbf{m L})\end{array}$ & $\begin{array}{c}\text { Índice de } \\
\text { Expansão }\end{array}$ & $\begin{array}{c}\text { Volume } \\
\text { Produzido } \\
(\mathbf{m L})\end{array}$ & $\begin{array}{c}\text { Fator de } \\
\text { expansão } \\
\left(\mathbf{c m}^{3}\right)\end{array}$ \\
\hline E01 & 2,5 & 1 & $3,08^{\mathrm{b}} \pm 0,03$ & $0,33^{\mathrm{c}} \pm 0,02$ & $1,44^{\mathrm{a}} \pm 0,01$ & $18,00^{\mathrm{ab}} \pm 0,57$ & $1,85^{\mathrm{c}} \pm 0,03$ \\
E02 & 4,1 & 1 & $4,41^{\mathrm{a}} \pm 0,02$ & $0,23^{\mathrm{a}} \pm 0,04$ & $1,50^{\mathrm{a}} \pm 0,01$ & $23,00^{\mathrm{d}} \pm 0,38$ & $2,12^{\mathrm{d}} \pm 0,08$ \\
E03 & 2,5 & 3 & $2,08^{\mathrm{c}} \pm 0,02$ & $0,47^{\mathrm{a}} \pm 0,03$ & $1,20^{\mathrm{b}} \pm 0,01$ & $9,00^{\mathrm{c}} \pm 0,24$ & $1,36^{\mathrm{e}} \pm 0,03$ \\
E04 & 4,1 & 3 & $4,14^{\mathrm{e}} \pm 0,08$ & $0,23^{\mathrm{a}} \pm 0,01$ & $1,50^{\mathrm{a}} \pm 0,01$ & $16,00^{\mathrm{a}} \pm 0,39$ & $1,56^{\mathrm{a}} \pm 0,04$ \\
E05 & 1,6 & 2 & $2,75^{\mathrm{b}} \pm 0,26$ & $0,43^{\mathrm{e}} \pm 0,12$ & $1,43^{\mathrm{a}} \pm 0,02$ & $8,30^{\mathrm{c}} \pm 0,64$ & $1,20^{\mathrm{b}} \pm 0,06$ \\
E06 & 5 & 2 & $4,08^{\mathrm{e}} \pm 0,04$ & $0,24^{\mathrm{a}} \pm 0,02$ & $1,31^{\mathrm{e}} \pm 0,03$ & $20,00^{\mathrm{b}} \pm 0,41$ & $1,89^{\mathrm{c}} \pm 0,01$ \\
E07 & 3,3 & 0 & $4,08^{\mathrm{e}} \pm 0,03$ & $0,24^{\mathrm{a}} \pm 0,01$ & $1,46^{\mathrm{a}} \pm 0,02$ & $29,00^{\mathrm{e}} \pm 0,57$ & $2,07^{\mathrm{d}} \pm 0,03$ \\
E08 & 3,3 & 4 & $2,61^{\mathrm{b}} \pm 0,06$ & $0,39^{\mathrm{d}} \pm 0,02$ & $1,10^{\mathrm{c}} \pm 0,02$ & $10,66^{\mathrm{c}} \pm 0,52$ & $1,20^{\mathrm{b}} \pm 0,02$ \\
E09 & 3,3 & 2 & $4,06^{\mathrm{e}} \pm 0,05$ & $0,28^{\mathrm{b}} \pm 0,01$ & $1,21^{\mathrm{b}} \pm 0,03$ & $18,66^{\mathrm{ab}} \pm 0,69$ & $1,55^{\mathrm{a}} \pm 0,05$ \\
E10 & 3,3 & 2 & $4,02^{\mathrm{e}} \pm 0,03$ & $0,28^{\mathrm{b}} \pm 0,02$ & $1,13^{\mathrm{cd}} \pm 0,03$ & $18,00^{\mathrm{ab}} \pm 0,33$ & $1,53^{\mathrm{a}} \pm 0,04$ \\
E11 & 3,3 & 2 & $4,08^{\mathrm{e}} \pm 0,04$ & $0,28^{\mathrm{b}} \pm 0,01$ & $1,25^{\mathrm{e}} \pm 0,03$ & $18,66^{\mathrm{ab}} \pm 0,44$ & $1,55^{\mathrm{a}} \pm 0,02$ \\
\hline
\end{tabular}

${ }^{1}$ Letras distintas em uma mesma coluna apresentaram diferenças significativas ao nível de $5 \%$ de significância $(\mathrm{p} \leq 0,05)$.

Quando a incorporação de sal foi de $6 \mathrm{~g}$ para uma quantidade de $5 \mathrm{~g}$ de fermento biológico seco, o que corresponde ao E05, houve prejuízo nas propriedades de expansão, o volume específico obtido foi de $2,75 \mathrm{~mL} / \mathrm{g}$, para uma densidade de $0,43 \mathrm{~g} / \mathrm{mL}$, apresentou baixo volume produzido $(8,30 \mathrm{~mL})$ e fator de expansão máximo, o que caracteriza pães de qualidade inferior. Para o E07, onde não houve adição de sal, apenas de $10 \mathrm{~g}$ de fermento biológico seco, o volume produzido foi o maior apresentado; 29,00 mL.

A figura 2 apresenta a superfície de resposta e a curva de contorno da ação do fermento biológico e do sal sobre o fator de expansão máximo das massas.
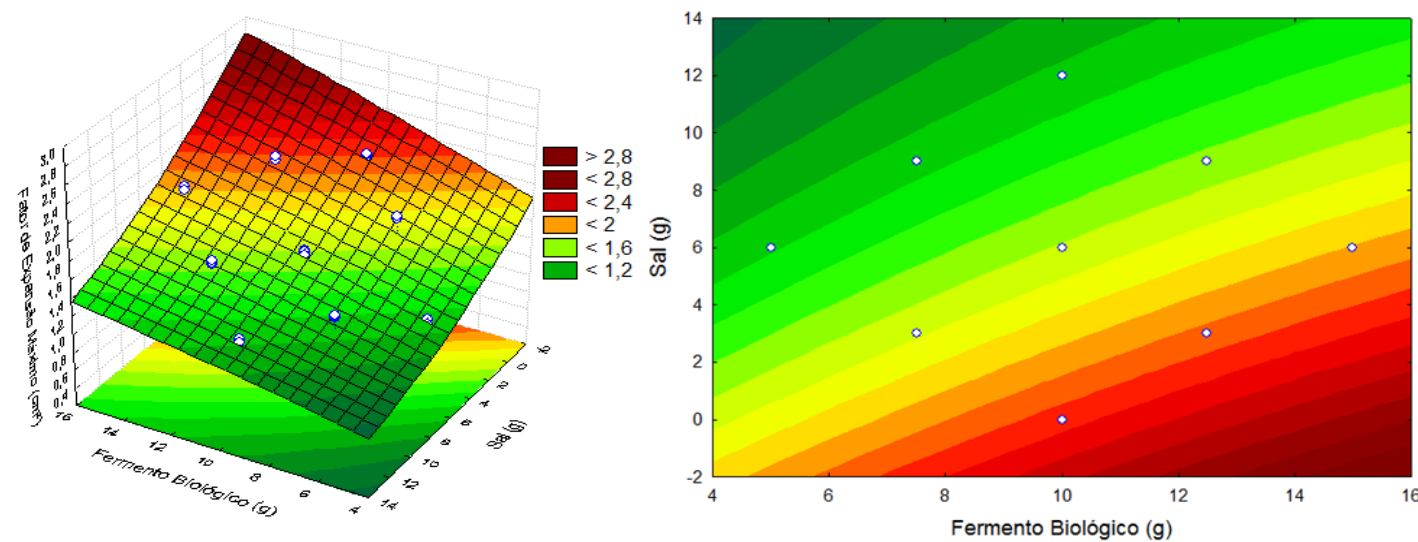

Figura 2 - Superfície de resposta, curva de contorno da ação do fermento biológico e do sal sobre o fator de expansão máximo da massas

Pode-se notar que quanto maior a quantidade de fermento empregada, maior foi o fator de expansão máximo das massas. Quando houve a adição de sal, ocorreu a redução deste parâmetro físico para valores inferiores a $2,00 \mathrm{~cm}^{3}$, mostrando que a fermentação ocorreu de forma lenta e dificultosa devido à função limitante de fermentação do sal. A região ótima 
apresentada consistiu na adição de $8 \mathrm{~g}$ de fermento biológico, sem a incorporação de sal até $16 \mathrm{~g}$ de fermento combinado com até $4 \mathrm{~g}$ de sal.

A figura 3 apresenta a superfície de resposta e curva de contorno da ação do fermento biológico e sal sobre o volume produzido das massas.
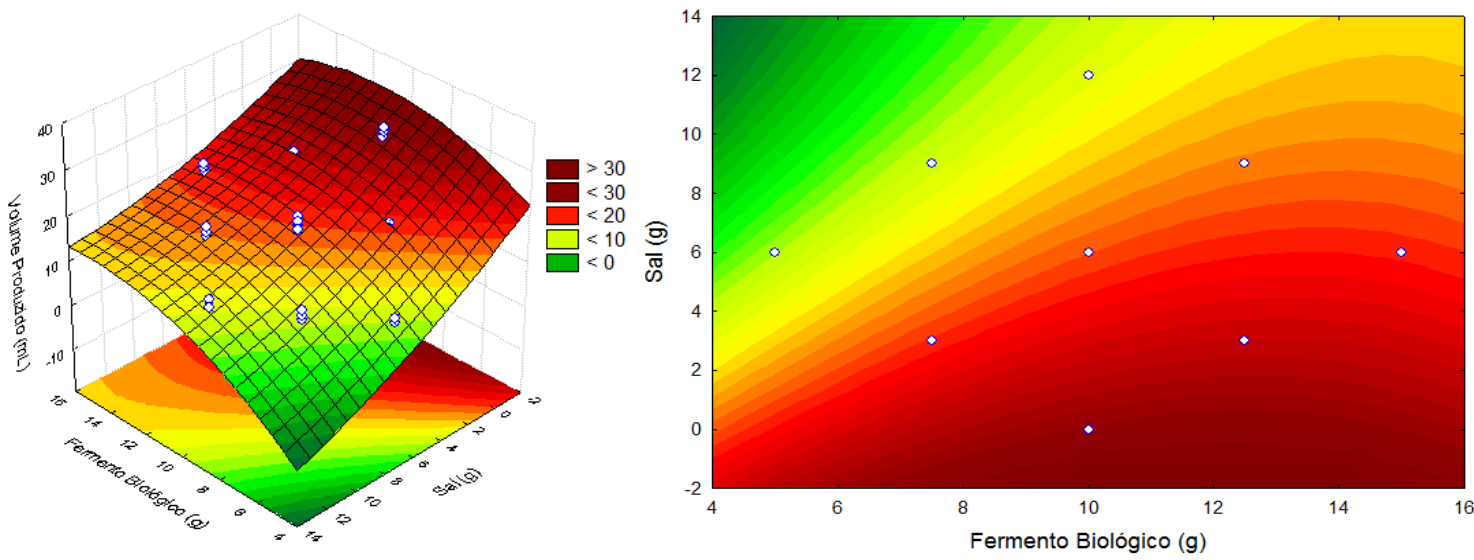

Figura 3 - Superfície de resposta, curva de contorno da ação do fermento biológico e do sal sobre o volume produzido das massas

Para o volume produzido, a ação danosa do sal ocorreu em menor proporção. Quantidades superiores a $4 \mathrm{~g}$, quando combinados com valores superiores a $10 \mathrm{~g}$ de fermento, os volumes produzidos foram superior a $20 \mathrm{~mL}$, caracterizando massas de boa expansão. A região otimizada compreendeu na incorporação de quantidades mínimas de sal e entre 8 e $16 \mathrm{~g}$ de fermento biológico, correspondendo a volumes superiores a $30 \mathrm{~mL}$.

A figura 4 fornece a superfície de resposta e curva de contorno da ação do fermento biológico e sal no volume específico dos pães.
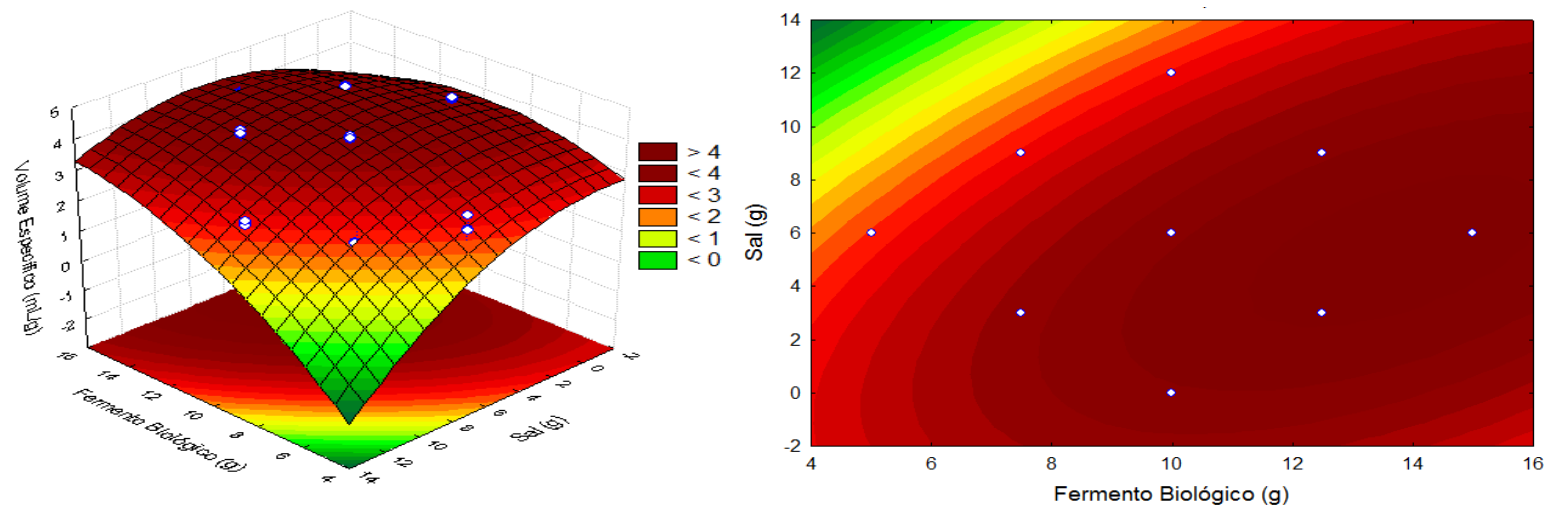

Figura 4 - Superfície de resposta, curva de contorno da ação do fermento biológico e do sal sobre o volume específico dos pães

O volume específico é uma importante propriedade física dos pães, uma vez que exerce forte influência na preferência do consumidor. A maximização do volume específico ocorreu para valores superiores a $4,00 \mathrm{~mL} / \mathrm{g}$, quando houve incorporação de 10 a $16 \mathrm{~g}$ de fermento biológico, combinado com adição de 1 a $10 \mathrm{~g}$ de sal. 
A figura 5 apresenta a superfície de resposta e a curva de contorno da ação do fermento biológico e do sal sobre a densidade dos pães tipo forma.
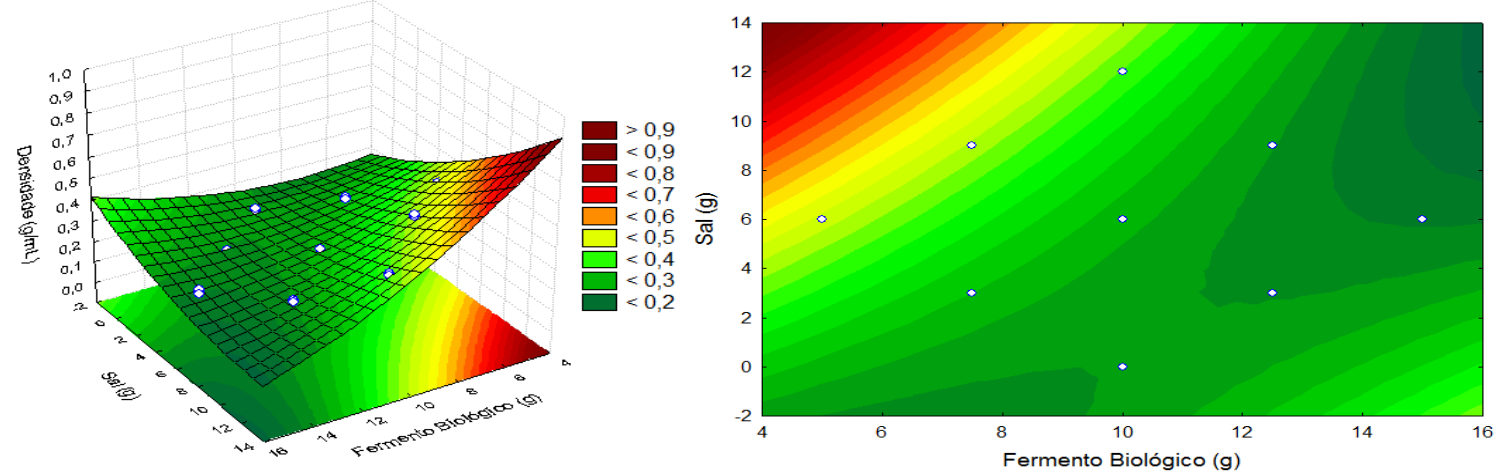

Figura 5 - Superfície de resposta e curva de contorno da ação do fermento biológico e do sal sobre a densidade dos pães

A região ótima para a variável resposta densidade compreendeu para valores inferiores a faixa de 0,2 a $0,3 \mathrm{~g} / \mathrm{mL}$, onde apresentou pequenas quantidades de sal, combinadas com quantidades de 6 a $8 \mathrm{~g}$ de fermento biológico ou elevadas quantidades de sal juntamente com grandes qualidades de fermento.

A figura 6 fornece a superfície de resposta e curva de contorno da ação do fermento biológico e do sal sobre o índice de expansão dos pães.
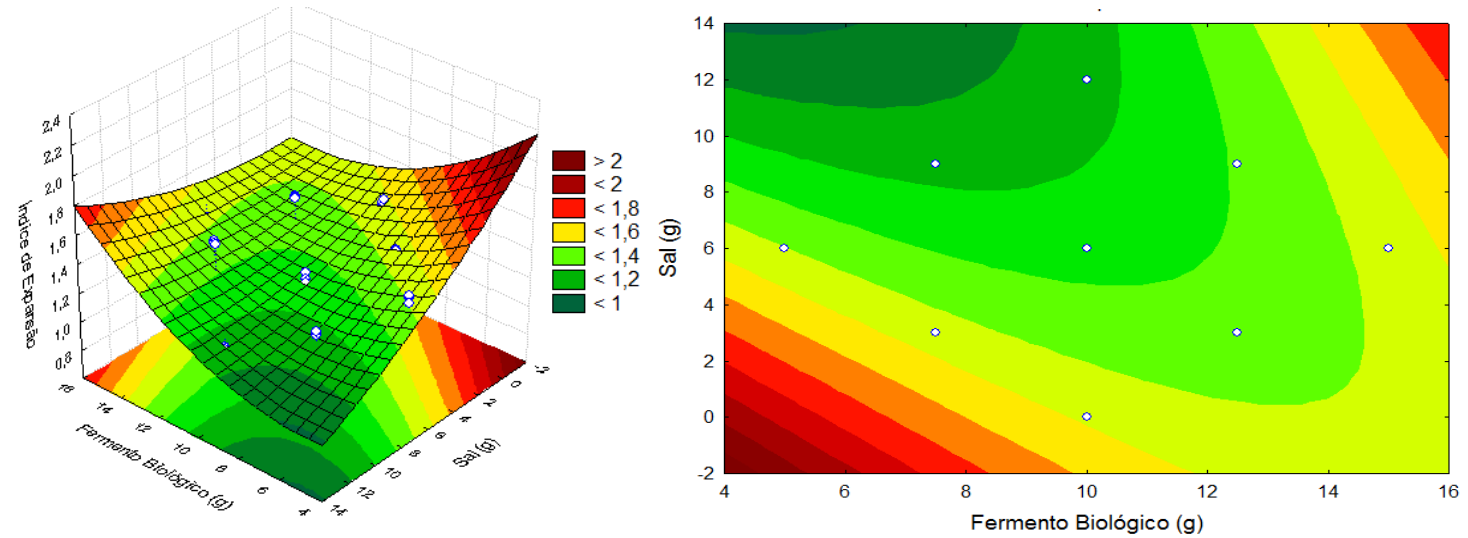

Figura 6 - Superfície de resposta e curva de contorno da ação do fermento biológico e do sal sobre o índice de expansão dos pães

A incorporação de quantidades superiores a $4 \mathrm{~g}$ de sal, independente da quantidade de fermento proporcionou índices de expansão inferiores a 1,60, entretanto, são valores satisfatórios para este parâmetro, superiores aos encontrados por Amaya et al., (2013).

\subsection{CONCLUSÃO}

Verificou-se que, independente da quantidade de fermento biológico empregada, o sal reduziu a capacidade de expansão das massas. As condições ótimas encontradas foram de incorporação do sal em até $2 \%$ e entre $3 \%$ e $5 \%$ para o fermento biológico, sem prejuízo significativo aos parâmetros físicos dos pães. 


\subsection{REFERÊNCIAS}

AMAYA, D. A. D.; PINHEIRO, G. K.; BRASIL, D. L.; MELO, P. E. F.; ZAMBELLI, R. A. Efeito do suco de caju na capacidade de expansão de massas durante o processo de fermentação. Magistra, v. 25, p. 482-487, 2013 a.

AMERICAN ASSOCIATION OF CEREAL CHEMISTS - A. A. C. C. Approved methods of American Association of Cereal Chemists. 9 ed. St. Paul: 1995.

CALLEJO, M. A. Present situation on the descriptive sensory analysis of bread. J. Sens. Stud., v. 26, p. 255-268, 2011.

GABRIC, D.; BEN-AISSA, F.; LE-BAIL, A.; MONTEAU, J. Y.; CURIC, D. Impact of process conditions on the structure of pre-fermented frozen dough. J. Food Eng. n.105, p. $361-366,2011$

GÓMEZ, M.; OLIETE, B.; PANDO, V.; RONDA, F.; CABALLERO, P. A. Effect of fermentation conditions on bread staling kinectics. Eur. Food Res. Technol., v. 226, p. 13791387, 2008.

KATINA, K.; HEINIO, R. L.; AUTIO, K.; POUTANEN, K. Optimization of sourdough process for improved sensory profile and texture of wheat bread. Food Sci. Technol., v. 39, p. 1189-1202, 2006.

LYNCH, E. J.; DAL BELLO, F.; SHEEHAN, E. M.; CASHMAN, K. D.; ARENDT, E. K. Fundamental studies on the reduction of salt on dough and bread characteristcs. Food. Res. Int., v. 42, p. 885-891, 2009.

OUERDANE, L.; ZÓLTAN, M. Production and characterization of fully selenomethioninelabeled Saccharomyces. J. Agric. Food. Chem., v. 56, p. 11792-11799, 2008.

PALOMBA, S.; BLAIOTTA, G.; VENTORINO, V.; SACCONE, A.; PEPE, O. Microbial charactarization of sourdough for sweet baked products in the Campania region (southern Italy) by a polyphasic approach. Ann. Microbiol., v. 61, p. 307-314, 2011.

RÓZYLO, R.; DZIKI, D.; LASKOWSKI, J. Changes in physical and the sensorial properties of wheat bread caused by interruption and slowing of the fermentation of yeast-based leaven. J. Cereal Sci., v. 59, p. 88-94, 2014.

SILVA, M. R.; GARCIA, G. K. S.; FERREIRA, H. F. Caracterização química, física e avaliação da aceitação de pão de queijo com baixo teor energético. Alim. e Nutr, v. 14, n. 1, p. 69-75, 2003. 